\section{A review of seismic wave attenuation} Luiz Fernando da Silva ${ }^{* 1}$, Webe João Mansur ${ }^{2}$, LAMEMO UFRJ ${ }^{1,2}$.

Copyright 2021, SBGf - Sociedade Brasileira de Geofísica

This paper was prepared for presentation during the $17^{\text {th }}$ International Congress of the Brazilian Geophysical Society held in Rio de Janeiro, Brazil, 16-19 August 2021.

Contents of this paper were reviewed by the Technical Committee of the $17^{\text {th }}$ International Congress of the Brazilian Geophysical Society and do not necessarily represent any position of the SBGf, its officers or members. Electronic reproduction represent any position of the SBGt, its officers or members. Electronic reproduction storage of any part of this paper for commercial purposes without the written consen of the Brazilian Geophysical Society is prohibited.

\section{Abstract}

Since the last century, research into the phenomenon of attenuation of seismic waves has been much discussed. This article describes a summarized version of the evolution of the study of attenuation mechanisms and their consequences and application in geophysics. A brief bibliographic review with examples and practical applications of the phenomenon of attenuation of seismic wave propagation is presented.

\section{Introduction}

The theory and application of seismic modeling are very relevant to the process of seismic interpretation and also in the development of inversion algorithms. The characteristics of the real materials of the layers that form the planet Earth are of anisotropy and anelasticity in general. It is known that the attenuation effects alter the amplitude and phase of recorded seismic waves. Seismic attenuation is an intrinsic property of rocks causing dissipation of energy as seismic waves propagate through the subsurface. It results in the decay of amplitude of the seismic waves. Attenuation is related to velocity dispersion. The energy of seismic waves is conserved only if it travels through a perfectly elastic medium. Propagating seismic waves loose energy due to: Geometrical spreading (wavefront radiating from a point source is distributed over a spherical surface of increasing size), Absorption (anelastic attenuation), Scattering (elastic attenuation). The quality factor $Q$ is a parameter that measures how much the medium attenuates seismic waves. 1/Q as a dissipation factor was introduced by Knopoff (Knopoff, 1958) and is similar to what was used in electrical circuit theory. Zener (Zener, 1958) was also one of the first to investigate hidden variables and memory effects similar to the theory of memory variables we have today and is the most common approach to viscoelastic equations (Carcione et al., 1988). The viscoelastic theory parameterized the $Q$ factor and is based on rheological models where each one presents unique aspects in the frequency domain (Liu et al., 1976; Kjartansson, 1979; Blanch et al. 1995; Moczo and Kristek, 2005; Moczo et al., 2007; Vasheghani FLR Lines, 2009; Carcione JM, 2014). The $Q$ factor is much discussed today and due to its complexity, its own taxonomy has been created in several applications (Igor Morozov and Amin Baharvand Ahmadi, 2015). The scientific community has been concerned for decades with the problem of incorporating the phenomenon of attenuation in the time domain modelling, since all laboratory research has shown a strong dependence on the frequency and the phenomenon of seismic wave propagation carries the entire frequency content.

\section{Viscoelastic Theory}

The mechanical response of a viscoelastic material body, subjected to stress variations, is non-instantaneous; and its time response characterizes its viscoelastic behavior. (Carcione J. M., 2014). The response of the medium to stress occurs with a delay due to the viscous behavior of the material: the material has memory. To interpret the viscoelastic behavior one usually uses springs and dashpots. The differential representation that describes the behavior of models consisting of springs (Hookeanas) and dampers (a viscous part Newtonian) (T. Alfrey and P. Doty, 1945; Christensen, RM, 1982) are given in equations 1 and 2 for elastic and viscous materials, respectively:

$\sigma_{\mathrm{ij}=\mathrm{C}_{\mathrm{jjk} \mid} \varepsilon_{\mathrm{k} l}} \quad$ (1) and $\sigma_{\mathrm{ij}}=\Psi_{\mathrm{ijkl} \mid} * \frac{\partial \varepsilon_{k l}}{\partial t} \quad$ (2), where $\Psi_{\mathrm{ijkl}}$ is relaxation-tensor.

In the Maxwell model, one spring and one dashpot are connected in series. In the Kelvin-Voigt model, one spring and one dashpot are connected in parallel and the Standard Linear Solid, considers two sprins and one dashpot (figure 1). The mechanism of evanescent memory (memory variables: Day and Minster, 1984; Emmerich and Korn, 1987) can be described by the equations below, where stresses are related in time with the strains and vice versa.

$\sigma(\mathrm{t})=\psi * \frac{\partial \varepsilon}{\partial \mathrm{t}}(3), \quad \varepsilon(\mathrm{t})=\mathrm{X} * \frac{\partial \sigma}{\partial \mathrm{t}}(\mathbf{4})$

where $\psi$ is the relaxation function and $X$ is the fluency function (Creep compliance).

From theory we can write:

$\sigma(\mathrm{t})=\mathrm{M}(\mathrm{t}) * \varepsilon(\mathrm{t}) \quad(5)$

and in the frequency domain $\sigma(w)=M(\omega) \varepsilon(\omega)$. Where $\frac{\partial \Psi(\mathrm{t})}{\partial \mathrm{t}}=\mathrm{M}(\mathrm{t})$ is the complex modulus and its Fourier transform is:

\section{Memory Variable}

Be $\psi(t)=f(t) H(t)$ where $H(t)$ is Heaviside step function

then $\sigma(\mathrm{t})=\psi(\mathrm{t}) \quad * \frac{\partial \varepsilon}{\partial t}=\frac{\partial \Psi(\mathrm{t})}{\partial t} * \boldsymbol{\varepsilon}(\mathbf{t})=\left(\left(\frac{\partial \mathbf{f}(\mathrm{t})}{\partial \mathrm{t}} \mathbf{H}(\mathbf{t})+\mathbf{f}(\mathbf{t}) \boldsymbol{\delta}\right) *\right.$ $\varepsilon)$.

$\sigma(\mathrm{t})=\Psi(\mathrm{t}) * \frac{\partial \varepsilon}{\partial t}=\frac{\partial \Psi(\mathrm{t})}{\partial t} * \boldsymbol{\varepsilon}(\boldsymbol{t})=\frac{\partial f(t)}{\partial t} \mathbf{H}(\mathbf{t}) * \boldsymbol{\varepsilon}+\mathbf{f}(\mathbf{0}) \boldsymbol{\varepsilon}=\mathbf{e}+$ $\mathbf{f}(\mathbf{0}) \varepsilon$.

Where $\mathrm{e}=\frac{\partial \boldsymbol{f}(\boldsymbol{t})}{\partial \boldsymbol{t}} \mathbf{H}(\mathbf{t}) * \boldsymbol{\varepsilon}$ and the e time derivative is

$\frac{\partial \mathbf{e}}{\partial \mathbf{t}}=\frac{\partial^{2} \mathbf{f}(\mathbf{t})}{\partial \mathbf{t}^{2}} \mathbf{H}(\mathbf{t}) * \boldsymbol{\varepsilon}+\frac{\partial \mathbf{f}(\mathbf{0})}{\partial \mathbf{t}} \boldsymbol{\varepsilon} .(6)$

This last equation results in the equations of memory variables of the viscoelastic models (Carcione et al., 1988). Each viscoelastic model has a specific $M(t) / M(\omega)$, 
which describes the model behavior in time/frequency, respectively. The usual modeling process goes toward the discretization of the convolution integral. The quality factor is defined:

$$
Q(\omega)=\frac{\operatorname{Re}[M(\omega)]}{\operatorname{Im}[M(\omega)]} .(7)
$$

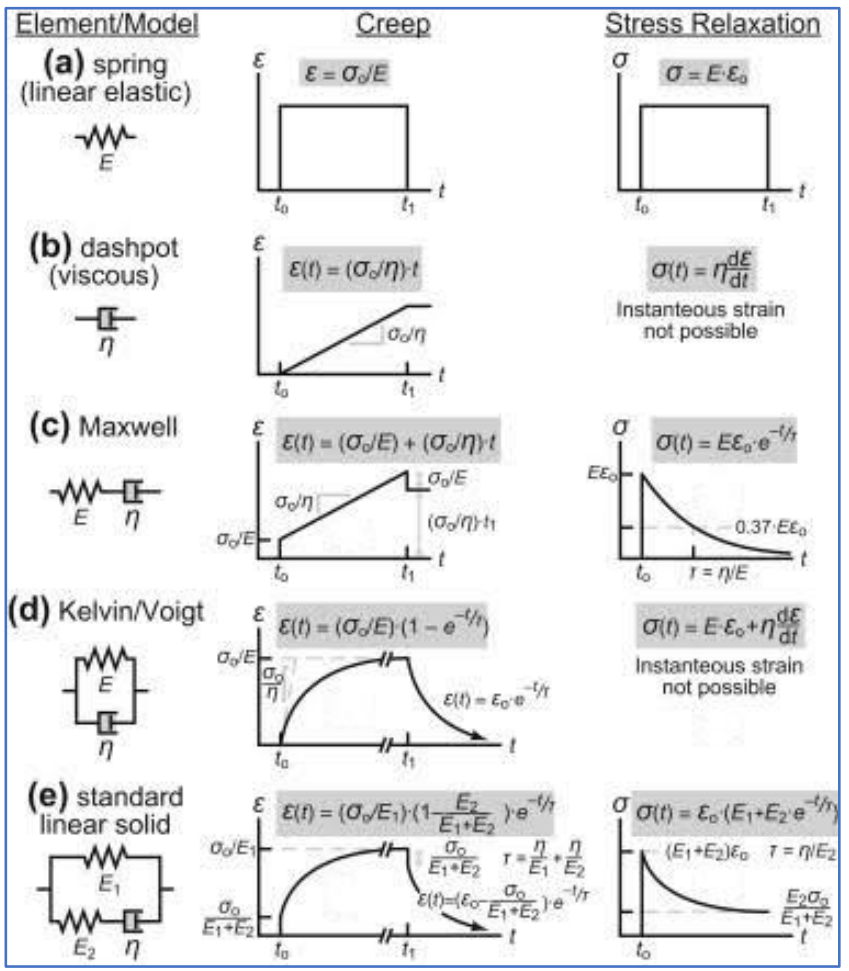

Figure 1: Maxwell, Kelvin-Voight and Standard Linear Solid (Viscoelasticity - an overview | ScienceDirect Topics sciencedirect.com).

\subsection{The Boltzmann Superposition Principle}

The Boltzmann superposition principle says that the effects of the mechanical history of linear materials are linearly additive. That is, a linear superposition of stresses leads to a corresponding linear superposition of deformations and vice versa. The mathematical formulation of this principle is the constitutive relationship between stress and strain given by a convolution integral and can be applied to the materials with linear behaviour (3), (4) and (5). The equations below briefly describe what was said earlier. Let it be an arbitrary linear superposition of strains and stresses given by:

$\boldsymbol{\varepsilon}(\mathbf{t})=\sum_{i=1}^{n} b_{i} \varepsilon_{i}(t)$ and $\boldsymbol{\sigma}(\mathbf{t})=\sum_{\mathbf{i}=\mathbf{1}}^{\mathbf{n}} \mathbf{b}_{\mathbf{i}} \boldsymbol{\sigma}_{\mathbf{i}}(t), \boldsymbol{b}_{\boldsymbol{i}}$ being an arbitrary constant independent of time.

From the convolution relation given in (5) and the distributive property of the operator, we have:

$$
\begin{gathered}
\sigma(t)=\sum_{\mathbf{i}=\mathbf{1}}^{\mathbf{n}} \mathbf{b}_{\mathbf{i}} \sigma_{\mathbf{i}}(t)=\sum_{\mathbf{i}=\mathbf{1}}^{\mathbf{n}} \mathbf{b}_{\mathbf{i}}\left(\mathbf{M} * \varepsilon_{\mathbf{i}}(\mathbf{t})\right)=\mathbf{M} * \\
\left(\sum_{\mathbf{i}=\mathbf{1}}^{\mathbf{n}} \mathbf{b}_{\mathbf{i}} \varepsilon_{\mathbf{i}}(t)\right) \cdot(6)
\end{gathered}
$$

It is seen that the response to the incremental load is independent of that due to other incremental loads. The individual sum of the responses, through a series of incremental loads, corresponds to the complete load history.

$\sigma(\mathrm{t})=\sum_{K=1}^{N} \Delta \sigma_{k}(t)$

\section{Method}

We used the theory of the mechanics of continuous media and a numerical simulation algorithm based on the finite difference method - FDM to simulate the propagation of waves (Virieux, J. (1986). The Maxwell model was used for the implementation of the algorithm. The method to incorporate attenuation through viscoelastic models is the memory variables in two-layer geologic site ( $\mathrm{P}$ wave). A two-layer geological model, $500 \mathrm{~m}$ deep and $500 \mathrm{~m}$ wide, was discretized with grid spacing $05 \mathrm{~m}$ in both vertical and horizontal directions. The wave propagation velocity of the compressional wave are $3000 \mathrm{~m} / \mathrm{s}$ and $2300 \mathrm{~m} / \mathrm{s}$ for the upper and lower layers respectively. Young's modulus, shear modulus, and Poisson coefficient are shown in figure 2. The shot position is at point $(250 \mathrm{~m}, 230 \mathrm{~m})$ and geophones are placed along the line $z=170 \mathrm{~m}$. The three cases analyzed considered frequency (of 1020 and $30 \mathrm{HZ}$ ) and a constant $Q$ factor equal to 10 and the behavior of a lowpass filter (Maxwell model) is verified according to seismograms in frequency. Results for the elastic case are also displayed so that one can see the representative change between simple elastic and viscoelastic analyses. In the two-layer geological model, the behavior of a lowpass filter (Maxwell model) is verified according to seismograms in frequency. The equation of motion in the elastic case was discretized in conjunction with the equation of memory variables (6).

$$
\boldsymbol{\rho} \frac{\mathbf{a}^{2} u_{i}}{\partial \mathbf{t}^{2}}=\nabla \cdot \sigma_{i j}+f_{i}(8)
$$

In this next example it is shown the effect of attenuation of the propagation of $\mathrm{SH}$ wave in a anisotropic viscoelastic media. We consider that the plane $(x, z)$ is the symmetrical plane of a monoclinic environment. Considering the transversal plane, it implies that the only non-zero deformations are the components $\sigma_{12}$ and $\sigma_{23}$.

Here are some steps to obtain the 3-D equation of motion, the formulation of the displacement of the $\mathrm{SH}$ motion equation is given by Euler Equation:

$\frac{\partial v_{2}}{\partial t}=\boldsymbol{\rho}^{-1}\left(\partial_{1} \sigma_{12}+\partial_{3} \sigma_{23}+f_{2}\right)$ 
The stress-strain relation:

$\sigma_{4}=c_{44} e_{4}+c_{46} e_{6}+c_{44} e_{23}$

$\sigma_{6}=c_{46} e_{4}+c_{66} e_{6}+c_{66} e_{12}$

Memory variable equations:

$\frac{\partial e_{23}}{\partial t}=\phi_{2}(0) e_{23}-\frac{1}{\tau_{\sigma}^{2}} e_{23}$

$\frac{\partial e_{13}}{\partial t}=\phi_{4}(0) e_{13}-\frac{1}{\tau_{\sigma} 4} e_{13}$

The simulation was performed in a two-layer geological model and the elastic constant $c_{46}$ is given as a function of the other two $c_{44}$ and $c_{66}$.

For more details on the wave equation that describes the behavior of the $\mathrm{SH}$ wave are found (Carcione J. M., 1997 and 2014).

\section{Results}

The results are described by the seismograms and seismic traces in the figures below and are in accordance with the theory described above. The results are in accordance with the theory.
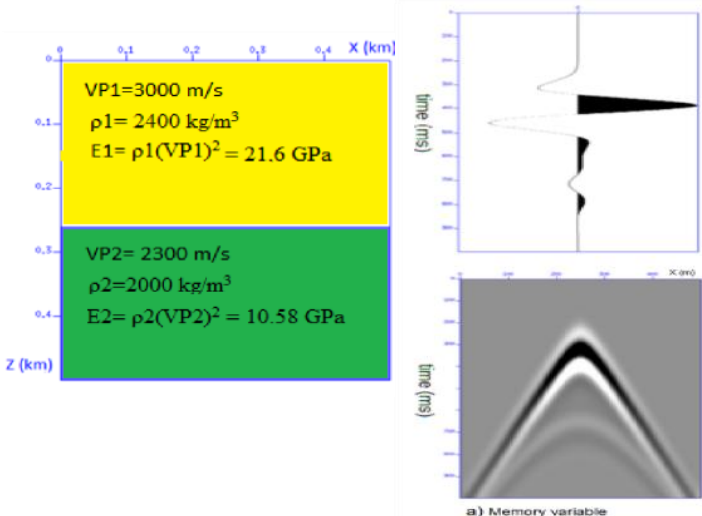

Figure 2: Sesimic Traces at $\mathrm{x}=250 \mathrm{~m}$ and Seismograms at the time $t=0.9 \mathrm{~s}$, source frequency $10 \mathrm{~Hz}$ and quality factor $Q=10$. a) memory variables.
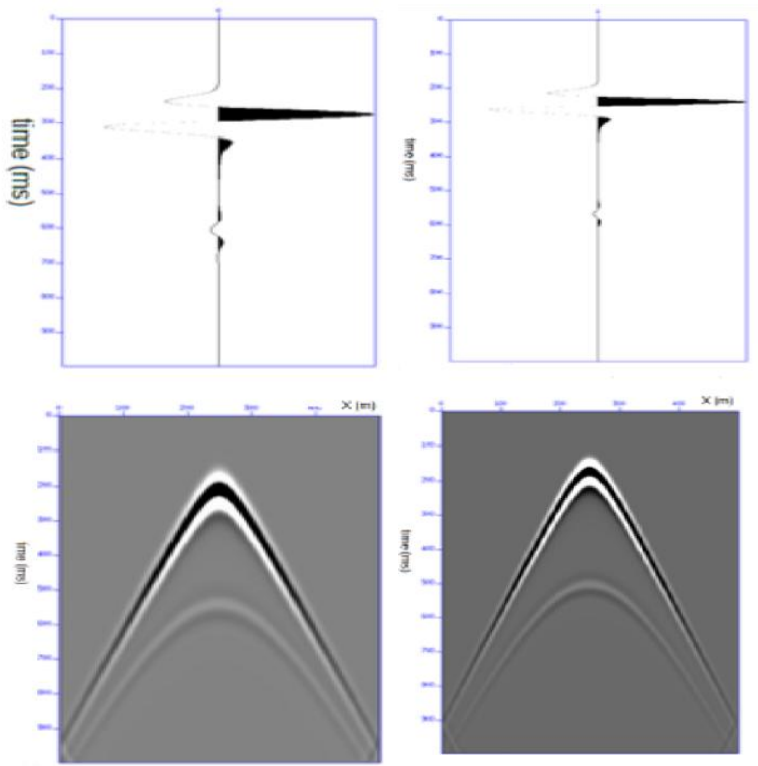

Figure 4: Seismic Traces in $\mathrm{x}=250 \mathrm{~m}$ and Seismograms at the time $t=0.9 \mathrm{~s}$, source frequency 20 and $30 \mathrm{~Hz}$ (respectively), quality factor $\mathrm{Q}=10$ (memory variables).

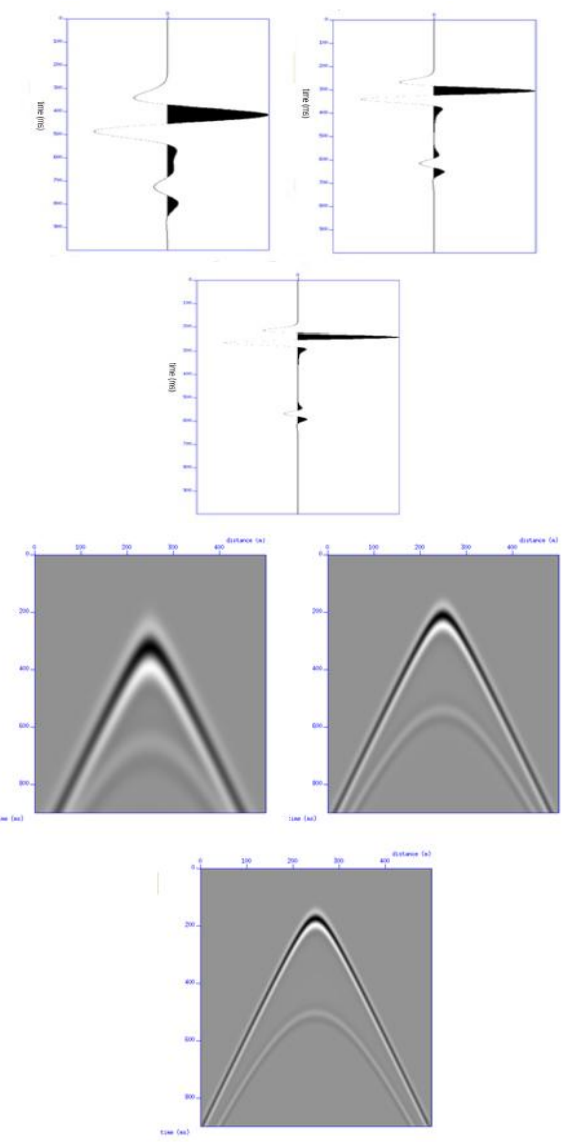

Figure 5: Seismic Traces in $\mathrm{x}=250 \mathrm{~m}$ (elastic case) and Seismograms at the time $\mathrm{t}=0.9 \mathrm{~s}$, source frequency 10 , 20 and $30 \mathrm{~Hz}$. 


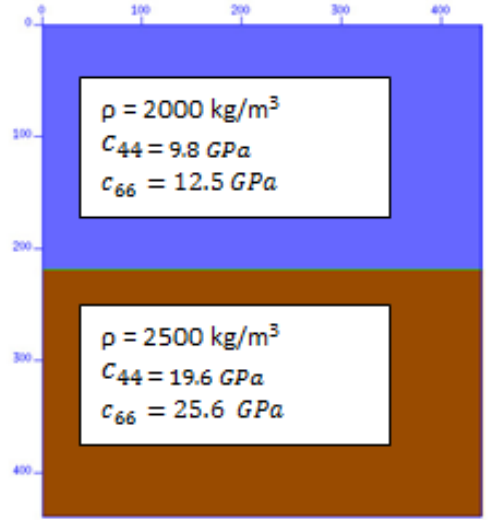

Figure 6: Two-layer geological model with 440 meters in both directions.

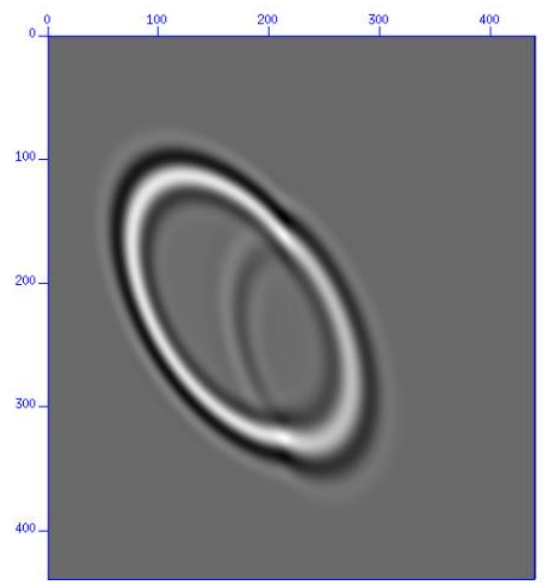

Figure 7: Snapshot - displacement (elastic case), time t= $0.3 \mathrm{~s}$, source frequency $15 \mathrm{~Hz}$.

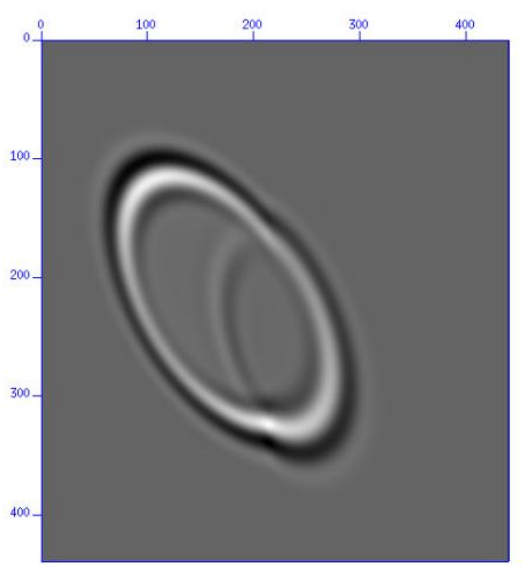

Figure 8: Snapshot - displacement (viscoelastic case), time $\mathrm{t}=0.3 \mathrm{~s}$, source frequency $15 \mathrm{~Hz}$

\section{Conclusions}

The incorporation of the attenuation of the medium in the propagation of seismic waves is very relevant in seismic and seismology. There is a wide variety of approaches to incorporate the phenomenon of attenuation in the propagation of seismic waves and their applications. This is still a problem where new theories may arise.

\section{Acknowledgments}

This study was financed in part by the Coordenacao de Aperfeicoamento de Pessoal de Nivel Superior-Brasil (CAPES) - Finance Code 001, CNPq (306.933/2014-4, 423.794/2016-7), Faperj: (E-26/203.021/2017, E26/010.001818/2015; E-26/010.101236/2018 and LAMEMO-UFRJ.

\section{References}

Blanch, J. O., J. O. A. Robertsson, and W. W. Symes, 1995, Modeling of a constant Q:

Methodology and algorithm for an efficient and optimally inexpensive viscoelastic technique:

Geophysics, 60, 176-184, doi: 10 .1190/1.1443744.

Carcione, J. M., D. Kosloff, and R. Kosloff, 1988, Viscoacoustic wave propagation simulation in the earth, 53, 769-777, Geophysics, doi:10.1190/1.1442512.

Carcione, J. M., D. Kosloff, and R. Kosloff, 1988, Wave propagation simulation in a linear viscoacoustic medium, Geophysical Journal International, 93, 393-401, doi: 10.1111/j.1365246X.1988.tb02010.x.

Carcione, J. M., D. Kosloff, and R. Kosloff, 1988, Wave propagation simulation in a linear viscoelastic medium, Geophysical Journal International, 95, 597-611, doi:10.1111/j.1365246X.1988.tb06706.x.

Carcione, J. M., 1993, Seismic modeling in viscoelastic media, Geophysics, 58, 110-120, doi:10.1190/1.1443340.

Carcione, J. M., 1997, Reflection and Refraction of Antiplane Shear Waves at a Plane Boundary between Viscoelastic Anisotropic Media, Proceedings of The Royal Society A Mathematical Physical and Engineering Sciences, 453(1960):919-942, doi: 10.1098/rspa.1997.0051

Carcione, J. M., 2014, Wavefields in real media, wave propagation in anisotropic, anelastic, porous and electromagnetic media: Elsevier Science.

Christensen, R. M., 1982, Theory of viscoelasticity - An introduction: Academic Press, Inc.

Day, S. M., and J. B. Minster, 1984, Numerical simulation of attenuated wavefields using a Pade approximant method: Geophysical Journal of the Royal Astronomical Society,78,105-118,doi:10.1111/j.1365-

246X.1984.tb06474.x.

Emmerich, H., and M. Korn, 1987, Incorporation of attenuation into time domain computations of seismic wave fields: Geophysics, 52, 1252- 1264, doi: 10.1190/1.1442386. 
Igor Morozov and Amin Baharvand Ahmadi, (2015), "Taxonomy of Q," GEOPHYSICS 80: T41-T49.

Kjartansson, E., 1979, Constant-Q wave propagation and attenuation, Journal of Geophysical Research, 84, B9, 4737-4748, doi: 10.1020/JB084iB09p04737.

Knopoff, L.,1958, Attenuation of small amplitude stress waves in solids, Reviews of Modern Physics, Volume 30, Number 4.

Liu, H.-P., D. L. Anderson, and H. Kanamori, 1976, Velocity dispersion due to anelasticity; implications for seismology and mantle composition,Geophysical Journal International, 47, 41-58, doi: 10.1111/j.1365246X.1976.tb01261.x.

Moczo, P., and J. Kristek, 2005, On the rheological models used for time-domain methods of seismic wave propagation, Geophysical Research Letters, 32, L01306, doi:10.1029/2004GL021598.

Moczo, P., J. Kristek, M. Galis, P. Pazak, and M. Balazovjech, 2007, The finite-difference and finiteelement modeling of seismic wave propagation and earthquake motion : Acta Phys.

Slovaca, 57(2), 177-406.

T. Alfrey, P. Doty, 1945, The Methods of Specifying the Properties of Viscoelastic Materials, Journal of Applied Physics, 16, 700, doi:10.1063/1.1707524.

Vasheghani F., and L. R. Lines, 2009, Viscosity and Q in heavy-oil reservoir characterization: The Leading Edge, 28, 856-860.

Virieux, J., 1986, P-SV wave propagation in heterogeneous media: velocity-stress finite difference method: Geophysics, 51, 889-901, doi:10.1190/1.1442147.

Zener, C., 1958, Anelasticity of metals, Nuovo

Cim 7, 544-568 (1958).

https://doi.org/10.1007/BF02751494SUOVO. 